\title{
ActiGait implantable drop foot stimulator in multiple sclerosis: a new indication
}

\author{
${ }^{*}$ K. Daniel Martin, MD, ${ }^{1}$ Witold H. Polanski, MD, ${ }^{1}$ Anne-Kathrin Schulz, ${ }^{1}$ Michael Jöbges, MD, PhD, ${ }^{2}$ \\ Tjalf Ziemssen, MD, PhD, ${ }^{3}$ Gabriele Schackert, MD, PhD, ${ }^{1}$ Thomas Pinzer, MD, ${ }^{1}$ and \\ Stephan B. Sobottka, MD' \\ ${ }^{1}$ Department of Neurosurgery and ${ }^{3}$ Center of Clinical Neuroscience, Department of Neurology, University Hospital \\ Carl-Gustav-Carus, Technical University of Dresden; and ${ }^{2}$ Neurologic Rehabilitation Centre, Brandenburg Klinik, \\ Bernau-Waldsiedlung, Germany
}

OBJECTIVE Direct stimulation of the peroneal nerve by the ActiGait implantable drop foot stimulator is a potent therapy that was described previously for stroke-related drop foot. The authors report here successful long-term application of the ActiGait implantable drop foot stimulator in patients with multiple sclerosis (MS).

METHODS Six patients with MS and 2 years of persisting central leg paresis received an implantable ActiGait drop foot stimulator after successful surface test stimulation. Ten weeks and 1 year after surgery, their gait speed, endurance, and safety were evaluated. Patient satisfaction was assessed with a questionnaire.

RESULTS In the 20-m gait test, stimulation with the ActiGait stimulator significantly reduced the time needed, on average, by approximately $23.6 \% 10$ weeks after surgery, and the time improved further by $36.3 \%$ after 1 year. The median distance covered by patients with the stimulator after 6 minutes of walking increased significantly from $217 \mathrm{~m}$ to $321 \mathrm{~m}$ and remained stable for 1 year; the distance covered by patients after surface stimulation was $264 \mathrm{~m}$. Patients with an implanted ActiGait stimulator noticed pronounced improvement in their mobility, social participation, and quality of life.

CONCLUSIONS The ActiGait implantable drop foot stimulator improved gait speed, endurance, and quality of life in all patients over a period of 1 year. It may serve as a new therapeutic option for patients with MS-related drop foot.

https://thejns.org/doi/abs/10.3171/2016.4.JNS1660

KEY WORDS ActiGait; multiple sclerosis; neuromodulation; peripheral nerve; drop foot; functional neurosurgery

$\mathrm{M}$ ULTIPLE Sclerosis (MS) is the most frequent chronic inflammatory disorder of the central nervous system. During the later phases of the disease, it may result in central paresis of the lower limbs with a drop foot, which can lead to an increased risk of falls, ${ }^{8}$ limited mobility, and a reduction in social participation and quality of life. Until now, using an ankle foot orthosis and surface stimulation of the peroneal nerve have been the only therapeutic options. However, increased walking speed and improved ankle and knee kinematics that led to improved quality of life ${ }^{1}$ were described recently after surface stimulation of the peroneal nerve. ${ }^{11,12,15,17}$ In 12 months of follow-up, this therapeutic effect was reported to be significant, ${ }^{16}$ and there is evidence that chronic use of the surface stimulator strengthens activation of the motor cortex and the descending connections. ${ }^{5}$ This might lead to an improvement in gait after long-term use,,$^{5}$ even when the stimulator is off.

Direct stimulation of the peroneal nerve by an implantable device was described recently as a new therapeutic option for patients with stroke-related drop foot, and it resulted in improved ankle joint kinematics and increased walking speed. ${ }^{2-4,10}$ The advantage of this system is the more specific stimulation. ${ }^{3}$ Patients who used this device also reported a better therapeutic effect and easier operability than patients who underwent surface stimulation. ${ }^{3}$ 
In addition, direct 4-channel nerve stimulation in 2 patients with MS-dependent drop foot was reported to result in improvements in walking speed in a follow-up time of 3 months. ${ }^{6}$ Although in patients after stroke and patients with MS, the resulting drop foot is caused by a central lesion, the therapeutic effects of nerve stimulation can differ over time, because MS may be progressive. In contrast, patients with MS tend to be younger and suffer less morbidity than patients after stroke, and they might experience a greater benefit from nerve stimulation and improvement in their quality of life.

In this study, we report the first, to our knowledge, successful long-term benefit of the ActiGait (Neurodan) implantable drop foot stimulator in 6 patients with MSrelated drop foot.

\section{Methods \\ Patient Screening}

Six patients with at least 1 year of stable MS and 2 years of persisting spastic paresis of the leg with a drop foot were offered a new therapeutic option via an implantable drop foot stimulator (patient characteristics are listed in Table 1). The drop foot was diagnosed according to foot during the swing phase. The possible benefit of an implantable stimulator was tested preoperatively by external functional surface stimulation of the peroneal nerve (CEFAR Step II [Compex Scandinavia AB], SN 2005-312, output $60 \mathrm{~mA}$, frequency $20-100 \mathrm{~Hz}$ ) with dorsiflexion of the ankle. This observational study was approved by the Technical University of Dresden ethics committee. The trial did not involve experimental medication, surgical products, or techniques.

\section{Gait Tests}

To evaluate possible changes in walking speed, patients were asked to walk $20 \mathrm{~m}$, and the time needed was documented. This test was performed preoperatively without walking aids, with an ankle orthosis, and with a surface stimulator and 10 weeks and 1 year after implantation of the ActiGait system. In each case, the patient was asked to perform the test at normal walking speed ("pleasant" gait) and as fast as possible ("maximal" gait). In addition, walking endurance was tested by having the patient walk for 6 minutes continuously before and after surgery. Finally, to assess the risk of falls, the time needed to stand up from a chair, walk $3 \mathrm{~m}$, and go back and be seated again was measured (Timed Up and Go test). ${ }^{14}$ All 3 tests were performed without any medical aids, with an ankle foot orthosis (tested only in the 20-m gait test), and with surface stimulation before the operation and with the activated ActiGait stimulator 10 weeks and 1 year after the operation. In addition, patient satisfaction was assessed with a subjective quality-of-life questionnaire (Table 2).

\section{Surgery}

For this study, the patients underwent implantation of the ActiGait system, which consists of an implantable 4-channel drop foot stimulator, an external control unit, a footswitch, and a clinical station ${ }^{2-4,9,10}$ (Fig. 1). MRI of features described by Perry et al., ${ }^{13}$ including a dragging

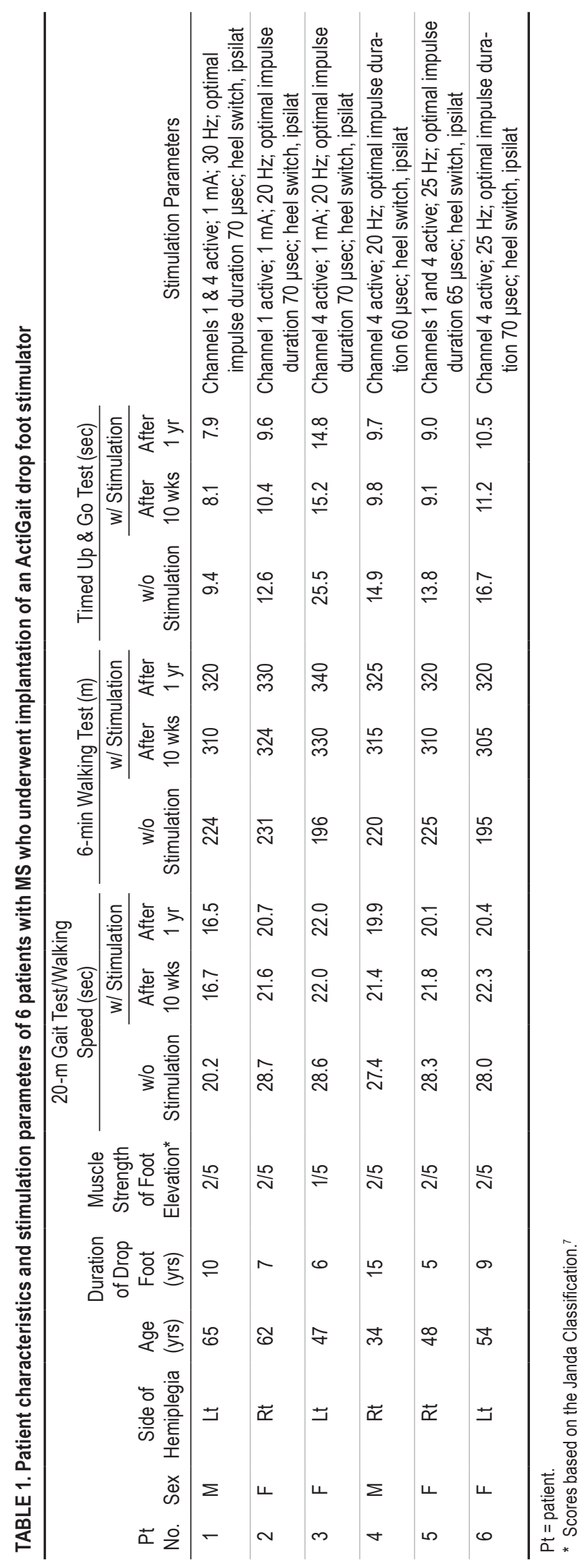


TABLE 2. Subjective quality-of-life survey from 6 patients with MS 1 year after implantation of an ActiGait drop foot stimulator

\begin{tabular}{|c|c|c|c|c|}
\hline Pt No. & $\begin{array}{c}\text { Have You Noticed Changes in Mobility } \\
\text { in Daily Living? (go for a walk, shopping, } \\
\text { public transit)* }\end{array}$ & $\begin{array}{l}\text { Have You Noticed Changes in } \\
\text { Interpersonal Contacts \& Social } \\
\text { Participation?† }\end{array}$ & $\begin{array}{l}\text { Would You } \\
\text { Recommend the } \\
\text { Operation? } \ddagger\end{array}$ & $\begin{array}{l}\text { Have You Noticed } \\
\text { Changes in Your } \\
\text { Quality Of Life?§ }\end{array}$ \\
\hline 1 & 3 & 3 & 1 & 2 \\
\hline 2 & 3 & 2 & 1 & 3 \\
\hline 3 & 2 & 3 & 1 & 2 \\
\hline 4 & 3 & 2 & 1 & 3 \\
\hline 5 & 2 & 3 & 1 & 3 \\
\hline 6 & 3 & 3 & 1 & 3 \\
\hline
\end{tabular}

the leg was performed preoperatively to determine the anatomical localization of the bifurcation of the sensible branch of the common peroneal nerve for proper electrode positioning. The operation was performed with the patient under general anesthesia, as described by Martin et al. ${ }^{9}$ In brief, the common peroneal nerve was exposed above the knee for $4 \mathrm{~cm}$, and dorsiflexion was tested using a nerve stimulator (GN 015). Afterward, the electrode cuff was closed around the nerve, and the stimulator body was fixed to the lateral femoral fascia. Finally, the correct positions of the cuff and the stimulator were controlled visually in maximal flexion and extension of the knee to ensure free gliding of the cuff.

\section{Postoperative Care and Programming of the Stimulator}

For the first 4 weeks after surgery, the patients had to restrict the movement of the leg on which surgery was performed to a maximal knee flexion of $30^{\circ}$. To avoid contractures during this time period, the Achilles tendon was stretched passively. Afterward, every channel was tested for the best dorsiflexion, and the stimulator was activated with $1 \mathrm{~mA}, 20-30 \mathrm{~Hz}$, and an optimal impulse duration of $70 \mu \mathrm{sec}$ (Table 1). Patients were able to readjust the impulse intensity with a programmer. Gait evaluation was performed after 10 weeks to measure gait speed and walking endurance, and the Timed Up and Go test was repeated. In addition, a subjective quality-of-life survey was given. All tests were repeated 1 year after surgery.

\section{Statistics}

The results of the gait tests are expressed as mean \pm SEM. The significance level was calculated with a MannWhitney U-test in Excel 2010 software.

\section{Results}

\section{Surgery and Postoperative Care}

Implantation of the ActiGait system in 6 patients with MS resulted in no technical or surgical complications. The implantable device did not appear bulky but was palpable in every patient. Programming of the ActiGait stimulator, as described above, was uncomplicated for all 6 patients. The programming time demand ranged between 45 and
60 minutes. Each patient described the handling of the ActiGait system in daily living as being uncomplicated and easy.

\section{Walking Speed (20-m Gait Test)}

The mean time to walk $20 \mathrm{~m}$ at normal walking speed was significantly $(\mathrm{p}=0.003)$ decreased from $25.9( \pm 4.4)$ seconds preoperatively without walking aids to $19.8( \pm$ 2.9) seconds with ActiGait stimulation 10 weeks after sur-

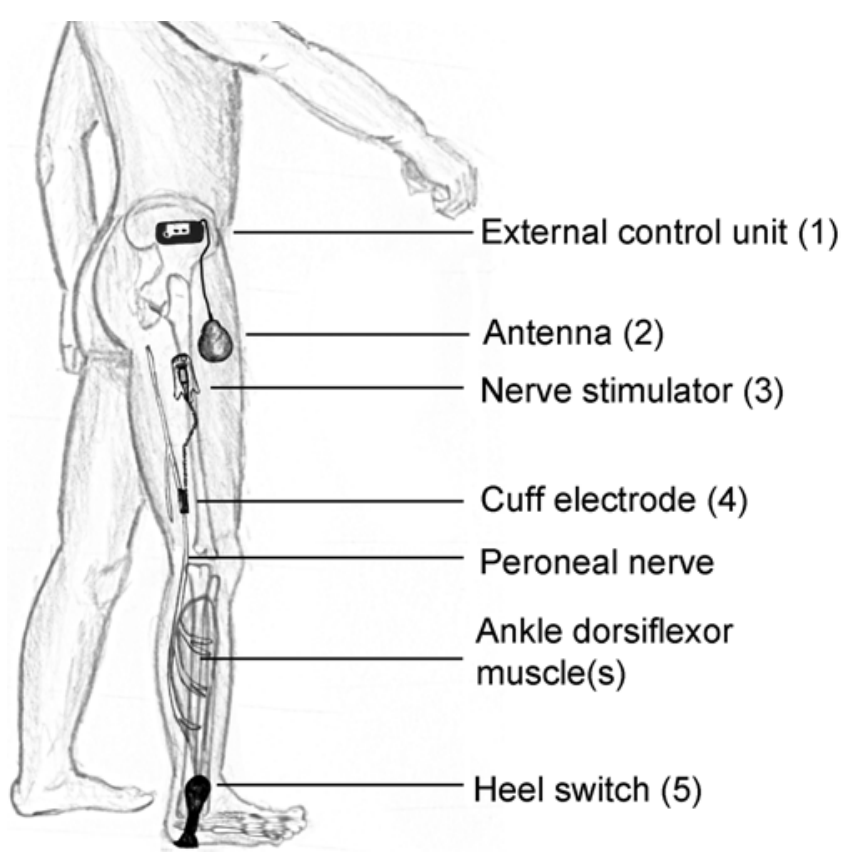

FIG. 1. Schematic illustration of the implantable and nonimplantable components of the ActiGait drop foot stimulator. The system consists of an external control unit (1) with a transmission coil (antenna) (2), which receives signals from the external footswitch (5) via a wireless radiofrequency signal. It enables precise activation depending on the gait cycle and allows for the adjustment of the stimulation parameters. The subcutaneously implanted stimulator (3) is connected to the 4-channel cuff (4), which is placed around the peroneal nerve. The stimulator communicates via a wireless radio frequency signal with the antenna and transmits the stimulation signals. Copyright Klaus Daniel Martin. Published with permission. 


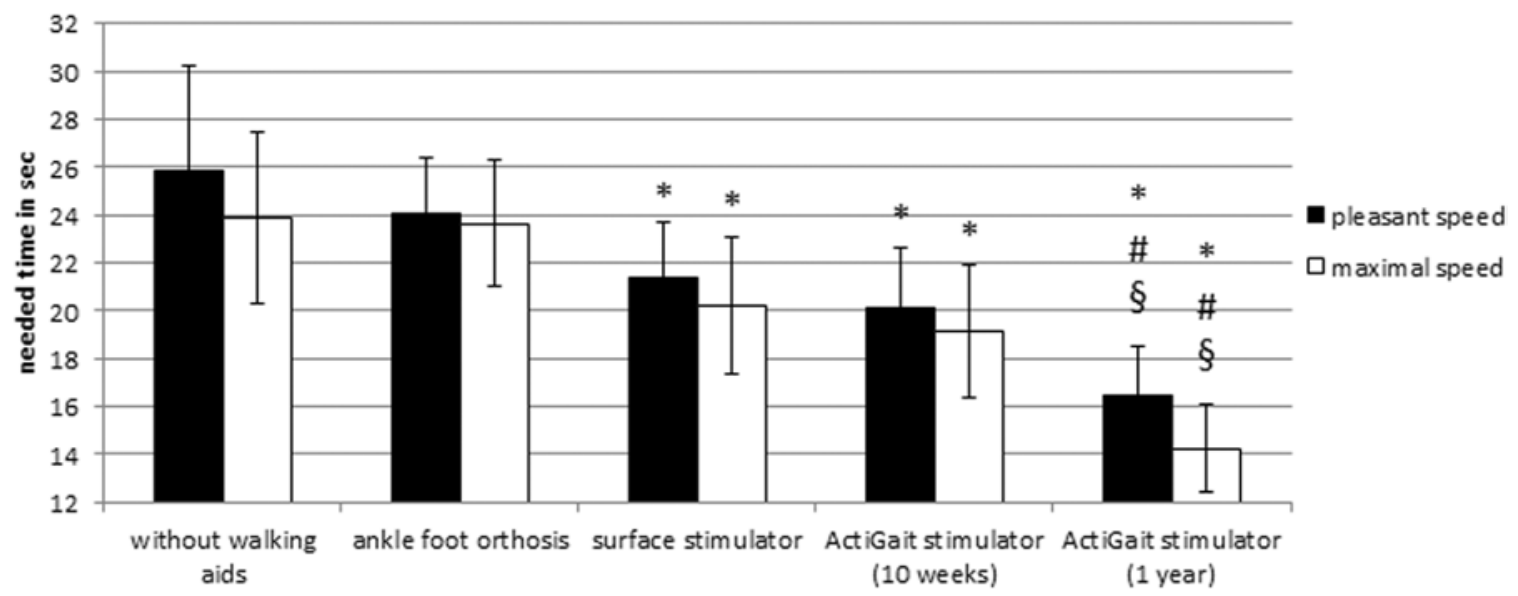

FIG. 2. Evaluation of mean walking speeds in the $20-\mathrm{m}$ gait test at pleasant and maximal walking speeds preoperatively and 10 weeks and 1 year after ActiGait implantation in 6 patients with MS. ${ }^{*} p<0.05$ compared with no stimulation; \#p $<0.05$ compared with use of ankle orthosis; $\$ p<0.05$ compared with surface stimulation.

gery and decreased further to $16.5( \pm 2)$ seconds 1 year after implantation. Surface stimulation also resulted in a significant $(\mathrm{p}<0.05)$ reduction in the mean time needed (to $21.4 \pm 2.3$ seconds). Ten weeks after surgery, there were no significant differences between ActiGait stimulation and surface stimulation. However, after 1 year, the ActiGait stimulator resulted in significant improvement over surface stimulation $(\mathrm{p}<0.05)$. In contrast, the use of an ankle orthosis resulted in no significant change in walking speed over that with no walking aids. Similar results were observed under maximal gait-speed conditions (Fig. 2). Video 1 exemplarily shows Patient 2 without walking aids and with ActiGait stimulation 1 year after surgery.

VIDEO 1. Clip showing walking speed of a patient with an activated ActiGait drop foot stimulator and no walking aids 1 year after surgery. Copyright Klaus Daniel Martin. Published with permission. Click here to view.

\section{6-Minute Walking Test}

Gait endurance was examined by measuring the distances that the patients walked within 6 minutes. Before stimulation, the patients walked, on average, $215 \pm 15.6 \mathrm{~m}$ in 6 minutes. With surface stimulation, the mean distance increased significantly $(\mathrm{p}<0.05)$ to $261 \pm 16 \mathrm{~m}$. After the patients underwent ActiGait implantation, their mean covered distance increased even further to $315 \pm 9.5 \mathrm{~m}$ 10 weeks after surgery and remained stable at $325 \pm 8 \mathrm{~m}$ 1 year after implantation (Fig. 3). This result, compared with those for patients without walking aids $(\mathrm{p}<0.05)$ and with surface stimulation $(\mathrm{p}<0.05)$, is significant.

\section{Timed Up and Go Test}

To assess activation time and the risk of falls, the Timed Up and Go test was performed without walking aids, with surface stimulation, and with ActiGait stimulation. The time needed for the test without walking aids was $19.5 \pm$ 6.8 seconds, which indicates mild mobility impairment (> 10 seconds needed). After surface stimulation, the time was reduced significantly to $13.2 \pm 3.3$ seconds. ActiGait stimulation 10 weeks after implantation led to a further reduction of the time needed to $9.29 \pm 1.2$ seconds, which was significant compared with surface stimulation, and a slight increase to $10.25 \pm 2.4$ seconds 1 year after surgery (Fig. 4).

\section{Subjective Quality-of-Life Survey}

For subjective changes in daily living after implantation of the ActiGait stimulator, the patients were asked to answer a short quality-of-life survey (Table 2). Four patients noticed an improvement in their mobility in daily living up to normal, in quality of life, and in social participation; 2 patients stated that they had a marked improvement. All of the patients said that they would recommend the surgical procedure.

\section{Discussion}

The aim of this study was to elucidate whether the ActiGait implantable drop foot stimulator can provide longterm improvements in gait speed and endurance and re-

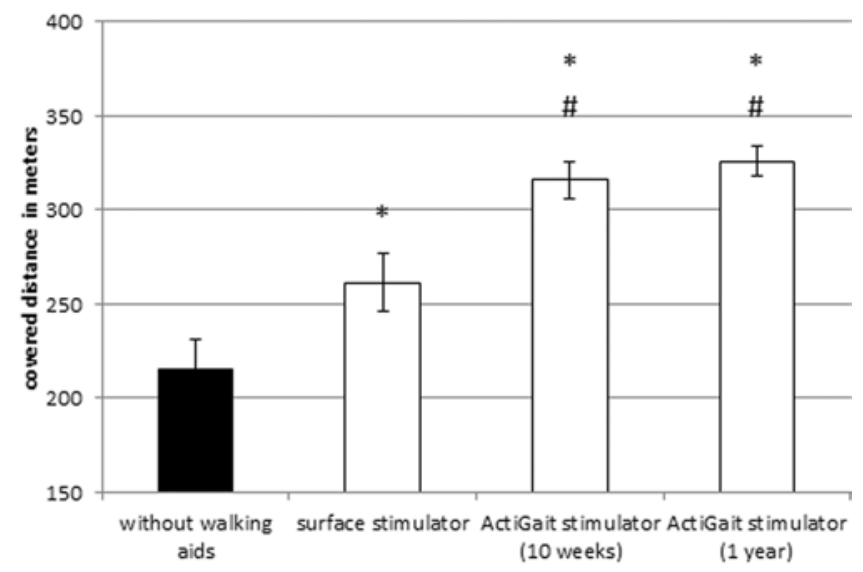

FIG. 3. Mean covered distances after 6 minutes of walking by 6 patients with MS with an ActiGait drop foot stimulator 10 weeks and 1 year after implantation. * $p<0.05$ compared with no stimulation; \#p $<0.05$ compared with surface stimulation. 


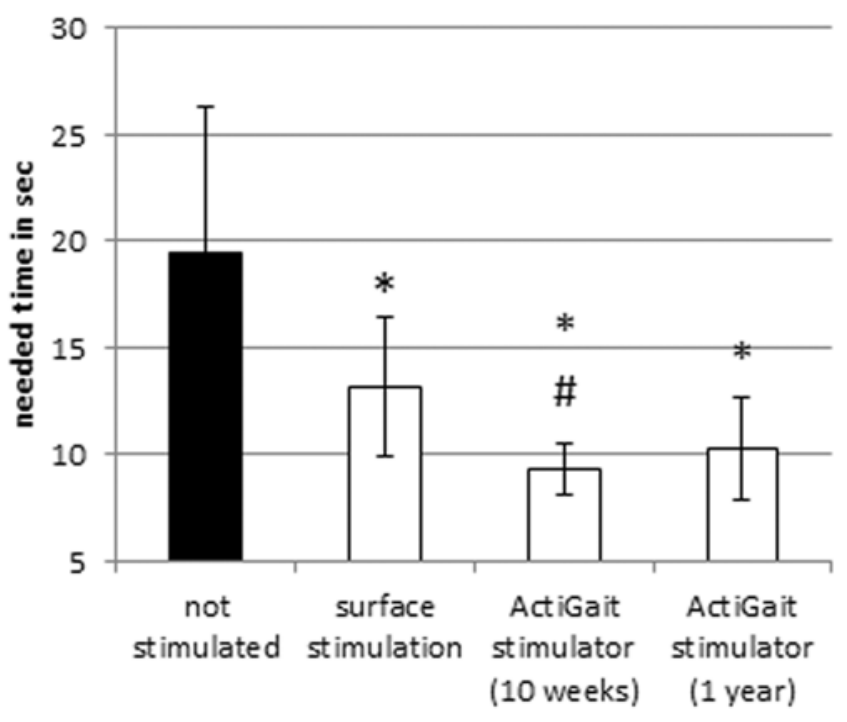

FIG. 4. Mean times needed to complete the Timed Up and Go tests by 6 patients with MS 10 weeks and 1 year after implantation of an ActiGait drop foot stimulator. ${ }^{*} p<0.05$ compared with no stimulation; \#p $<0.05$ compared with surface stimulation.

duce the risk of falls in patients with MS-related drop foot, because its efficacy and superiority over surface stimulation in patients after stroke were reported recently. ${ }^{10,16} \mathrm{In}$ this study, no adverse events were observed. Until now, only rare complications, such as hematoma and lymphedema (both in 1 of 5 patients), ${ }^{16}$ wound-healing deficit ( 1 of 15 patients) and infections ( 2 of 15 patients), ${ }^{3}$ and injury to the peroneal nerve ( 2 of 27 patients), ${ }^{10}$ have been reported. The surgery and postoperative care were easy and uncomplicated. The patients had no problems using the ActiGait stimulator in daily living. In addition, recent reports have shown that the implantable device is easier to use than the surface stimulator. ${ }^{3}$

Gait evaluations revealed that walking speed was increased significantly by $30.8 \% 10$ weeks after using the ActiGait system and increased more significantly by $57.0 \% 1$ year after surgery. These results are comparable with recent findings in patients after stroke, in which ActiGait stimulation resulted in improvements of $19 \%{ }^{4}$ and $25 \%{ }^{10}$ in a $10-\mathrm{m}$ gait test and $47 \% \%^{10}$ in a $20-\mathrm{m}$ gait test. In our study, the implantable stimulator was superior to an ankle foot orthosis $(\mathrm{p}=0.0173)$ and to surface stimulation after 10 weeks, but the difference was not significant ( $\mathrm{p}=$ 0.07672 ) . However, after 1 year, a significant improvement in gait over that with surface stimulation $(\mathrm{p}=0.0027)$ and with the foot orthosis $(p=0.00042)$ was found.

In addition, gait endurance was improved significantly by $47 \% 10$ weeks after surgery and increased further to $52 \%$ improvement after 1 year. Compared with surface stimulation, which had already improved gait endurance, ActiGait stimulation resulted in a pronounced and significant increase in the distance covered by our patients in the 6-minute walking test, and the therapeutic effect was stable over a period of 1 year. This result confirms the superiority of the implantable stimulator over surface stimulation. These findings might be explained by the fact that an implantable stimulator provides more specific stimulation with finer adjustability of stimulation parameters than does surface stimulation, and it produces a considerable orthotic effect with more physiological kinematics in the lower limb. ${ }^{16}$

Furthermore, in the Timed Up and Go test 10 weeks after surgery, a nonsignificant improvement of $47 \%$ was measured for patients with the ActiGait stimulator as a result of a safer gait. One year after implantation, this effect decreased to $43 \%$ but remained higher than the improvement with surface stimulation (29\% improvement compared with no walking aids). In this test, a normal gait without increased risk of falls is determined by a time needed of less than 10 seconds..$^{14}$ Only with ActiGait stimulation was this criterion reached, with $9.3( \pm 0.9)$ seconds needed to finish the test.

Finally, all 6 patients noticed at least a marked improvement of mobility in daily living, in quality of life, and in social participation, and they had no regrets about the implantation.

\section{Conclusions}

The ActiGait implantable drop foot stimulator is a safe and simple device for drop foot caused by a lesion of the central nervous system. It improved gait speed and endurance and quality of life and reduced the risk of falls in all 6 patients with MS. In addition, these results were stable and even improved further over a period of 1 year. These results are comparable with recent reported improvements in patients after stroke.

\section{Acknowledgments}

We thank Andrei Patriciu for excellent technical help during the surgical procedures and programming of the ActiGait stimulator and Prof. Jens Haase for scientific discussions.

\section{References}

1. Barrett C, Taylor P: The effects of the Odstock drop foot stimulator on perceived quality of life for people with stroke and multiple sclerosis. Neuromodulation 13:58-64, 2010

2. Burridge JH, Haugland M, Larsen B, Pickering RM, Svaneborg N, Iversen HK, et al: Phase II trial to evaluate the ActiGait implanted drop-foot stimulator in established hemiplegia. J Rehabil Med 39:212-218, 2007

3. Burridge JH, Haugland M, Larsen B, Svaneborg N, Iversen $\mathrm{HK}$, Christensen PB, et al: Patients' perceptions of the benefits and problems of using the ActiGait implanted drop-foot stimulator. J Rehabil Med 40:873-875, 2008

4. Ernst J, Grundey J, Hewitt M, von Lewinski F, Kaus J, Schmalz T, et al: Towards physiological ankle movements with the ActiGait implantable drop foot stimulator in chronic stroke. Restor Neurol Neurosci 31:557-569, 2013

5. Everaert DG, Thompson AK, Chong SL, Stein RB: Does functional electrical stimulation for foot drop strengthen corticospinal connections? Neurorehabil Neural Repair 24:168-177, 2010

6. Hausmann J, Sweeney-Reed CM, Sobieray U, Matzke M, Heinze HJ, Voges J, et al: Functional electrical stimulation through direct 4-channel nerve stimulation to improve gait in multiple sclerosis: a feasibility study. J Neuroeng Rehabil 12:100, 2015

7. Janda DH, Geiringer SR, Hankin FM, Barry DT: Objective evaluation of grip strength. J Occup Med 29: 569-571, 1987 
8. Luukinen H, Koski K, Laippala P, Kivelä SL: Predictors for recurrent falls among the home-dwelling elderly. Scand J Prim Health Care 13:294-299, 1995

9. Martin KD, Polanski W, Schackert G, Sobottka SB: New therapeutic option for drop foot with the ActiGait peroneal nerve stimulator-a technical note. World Neurosurg 84:2037-2042, 2015

10. Martin KD, Polanski WH, Schulz AK, Jöbges M, Hoff H, Schackert G, et al: Restoration of ankle movements with the ActiGait implantable drop foot stimulator: a safe and reliable treatment option for permanent central leg palsy. J Neurosurg 124:70-76, 2016

11. Miller L, Rafferty D, Paul L, Mattison P: A comparison of the orthotic effect of the Odstock dropped foot stimulator and the Walkaide functional electrical stimulation systems on energy cost and speed of walking in multiple sclerosis. Disabil Rehabil Assist Technol 10:482-485, 2014

12. Paul L, Rafferty D, Young S, Miller L, Mattison P, McFadyen A: The effect of functional electrical stimulation on the physiological cost of gait in people with multiple sclerosis. Mult Scler 14:954-961, 2008

13. Perry J, Garrett M, Gronley JK, Mulroy SJ: Classification of walking handicap in the stroke population. Stroke 26:982989, 1995

14. Podsiadlo D, Richardson S: The timed "Up \& Go": a test of basic functional mobility for frail elderly persons. J Am Geriatr Soc 39:142-148, 1991

15. Scott SM, van der Linden ML, Hooper JE, Cowan P, Mercer TH: Quantification of gait kinematics and walking ability of people with multiple sclerosis who are new users of functional electrical stimulation. J Rehabil Med 45:364-369, 2013

16. Stein RB, Everaert DG, Thompson AK, Chong SL, Whittaker M, Robertson J, et al: Long-term therapeutic and orthotic effects of a foot drop stimulator on walking performance in progressive and nonprogressive neurological disorders. Neurorehabil Neural Repair 24:152-167, 2010
17. Taylor P, Barrett C, Mann G, Wareham W, Swain I: A feasibility study to investigate the effect of functional electrical stimulation and physiotherapy exercise on the quality of gait of people with multiple sclerosis. Neuromodulation 17:7584,2014

\section{Disclosures}

The authors report no conflict of interest concerning the materials or methods used in this study or the findings specified in this paper.

\section{Author Contributions}

Conception and design: Martin, Polanski. Acquisition of data: Martin, Polanski, Schulz. Analysis and interpretation of data: Martin, Polanski, Schulz, Jöbges. Drafting the article: Martin, Polanski, Sobottka. Critically revising the article: Polanski, Ziemssen, Schackert, Pinzer, Sobottka, Jöbges. Reviewed submitted version of manuscript: Martin, Polanski, Schackert, Pinzer. Approved the final version of the manuscript on behalf of all authors: Martin. Statistical analysis: Polanski. Administrative/ technical/material support: Martin, Ziemssen, Schackert. Study supervision: Sobottka.

\section{Supplemental Information \\ Videos \\ Video 1. https://vimeo.com/165306345.}

\section{Correspondence}

K. Daniel Martin, Department of Neurological Surgery, CarlGustav-Carus University Hospital of the Technical University of Dresden, Fetscherstrasse 74, Dresden 01307, Germany. email: kontakt@dr-daniel-martin.com. 